IN S T I T U T O

$\mathrm{DE}$

M E D I C I N A

T R O P I C A L

$\mathrm{DE}$

SÃO PAULO

JOURNAL OF THE SÃO PAULO INSTITUTE OF TROPICAL MEDICINE

Universidad de la Sabana, Chía, Colombia

${ }^{2}$ Pontificia Universidad Javeriana, Bogotá, Colombia

${ }^{3}$ Hospital Universitario de La Samaritana, Bogotá, Colombia

Correspondence to: Diego Fernando Severiche-Bueno

Universidad de La Sabana, Campus Puente del Común, KM 7.5 Autopista Norte de Bogotá, Chía, Colombia

Tel: +571 861-5555, ext. 23342

E-mail: diegosebu@unisabana.edu.co

Received: 27 January 2021

Accepted: 12 April 2021

\section{Subarachnoid racemose neurocysticercosis with cerebellar involvement: an old friend in an infrequent location?}

\author{
Diana Maritza Parra-Cárdenas ${ }^{(1)}$, María Teresa Vargas-Cuervo ${ }^{(12}$, Jorge \\ Armando Montejo-Coy ${ }^{10}$, Carlos Mauricio Calderon-Vargas ${ }^{3}$, Diego \\ Fernando Severiche-Bueno 1
}

\section{ABSTRACT}

Taenia solium is the most common parasitic infection of the central nervous system and it can cause parenchymal or extra-parenchymal lesions. Subarachnoid cysticercosis is a type of extra-parenchymal infection in which the prevalence is not known and racemose NC with cerebellar involvement has been rarely reported. The diagnosis is challenging because of its similarity to other infectious diseases or to subarachnoid involvement of systemic malignancies. Treatment usually requires cysticide drugs, however, there are no randomized studies concerning the anti-parasitic treatment in subarachnoid NC. We present a case of racemose $\mathrm{NC}$ in the cerebellar hemisphere to draw attention to this pathology, endemic in many parts of the world; and highlight all the current gaps in our understanding of this entity.

KEYWORDS: Neurocysticercosis. Racemose form. Cerebellum. Central nervous system. Parasitic infections. Taenia solium.

\section{INTRODUCTION}

Neurocysticercosis (NC) is the most common parasitic disease of the central nervous system (CNS) worldwide. Cysticercosis is endemic in most Latin American countries, large regions of Asia including the Indian subcontinent, most of Southeast Asia and China, sub-Saharan Africa, and some countries of Eastern Europe ${ }^{1}$, with most cases coming from rural areas and with an overall prevalence of $8.55 \%$ in Colombia $^{1-5}$. After accidental ingestion of Taenia solium eggs (oncospheres), cysticerci hatch in the small intestine, penetrate into the intestinal wall and spread to other tissues through the bloodstream ${ }^{4}$. Although the majority of parasites are destroyed by the immune system, some can reach the CNS and allude the host's immune response in part due to the protection offered by the blood-brain barrier. Once in the CNS, they can develop to the larval stage. An inflammatory response usually occurs which is more intense after the larval degeneration ${ }^{1-4}$.

$\mathrm{NC}$ is subdivided by its location into parenchymal or extra-parenchymal forms, the latter being the less frequent. Extra-parenchymal neurocysticercosis involves infection of the subarachnoid space, the spinal cord (except in the rare case of intramedullary NC) or the ventricular system ${ }^{3,5,6}$. The prevalence of subarachnoid $\mathrm{NC}$ is not known, and high variability exists in the few studies in the literature ${ }^{7}$. In addition, racemose $\mathrm{NC}$ with cerebellar involvement has been rarely reported ${ }^{8,9}$. We present a case of racemose $\mathrm{NC}$ in the cerebellar hemisphere to draw attention to this pathology, endemic in many parts of the world; and highlight all the current gaps in our understanding of this entity. 


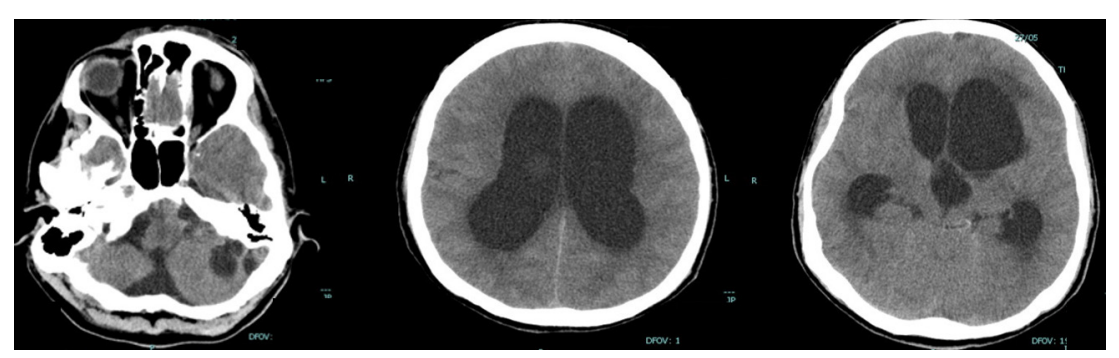

Figure 1 - Head CT. Severe dilation of the ventricular system, involving the third ventricle and lateral ventricles with transependymal edema and a multicystic lesion located in the subarachnoid space at the level of the lateral surface of the left cerebellar hemisphere.

\section{CASE REPORT}

A 51-year-old male patient, who lives in a rural area, was first taken to the emergency department of another hospital with a 3-month history of headaches associated with gait alterations due to weakness of the lower limbs, instability, difficulty to climb stairs without support, memory loss and behavioral changes. The initial computed tomography (CT) of the head, according to his medical record and the radiology report, did not reveal any evident lesion and a lumbar puncture was performed showing hyperproteinorrachia, hypoglycorrhachia and mild lymphocytic pleocytosis. However, fungal and bacterial cultures, cryptococcus latex agglutination test, Mycobacterium tuberculosis (MT) polymerase chain reaction and MT culture were negative. Due to the lymphocytic pleocytosis and the high prevalence of tuberculosis in our country, empiric anti-tuberculosis treatment was started.

Nevertheless, the patient was evaluated again three months after his initial visit due to the persistence of symptoms and the onset of urinary incontinence, drowsiness, emesis and fever. The physical examination revealed that the patient was disoriented in time and space and had short-term memory difficulties. Acalculia was noticed. The examination of the eye fundus revealed bilateral papilledema without any other pathological finding. Physical examination did not reveal tremors or asymmetric pronation of hands. Rapid alternating movements and finger-to-nose movements were normal. However, the pull test was positive, and an ataxic gait was noticed. Meningeal signs were absent. Blood tests were carried out showing leukocytosis and neutrophilia without eosinophilia and a new lumbar puncture was performed with similar results to the previous, once again, all the infectious diseases investigations were negative. Due to the lack of response to the initial treatment, the diagnosis of meningeal tuberculosis was ruled out and treatment was suspended. Serological tests for Histoplasma, Cryptococcus and Aspergillus were negative.

A head CT scan revealed ventricular dilation and the presence of a cerebellar cyst (Figure 1); the magnetic resonance imaging (MRI) of the brain showed a left cerebellar cyst along with severe dilation of the ventricular system compatible with hydrocephalus (Figure 2), so that a ventriculoperitoneal shunt was performed. Due to the clinical deterioration of the patient and the lack of a clear diagnosis, a biopsy of the cyst was performed with evidence of a cysticercus cyst (Figure 3). Racemose neurocysticercosis with cerebellar involvement was considered and $8 \mathrm{mg}$ of intravenous dexamethasone every $8 \mathrm{~h}$ in conjunction with albendazole at the maximum dose $(30 \mathrm{mg} / \mathrm{kg} /$ day/) was started, as Praziquantel is not commercially available in Colombia. While hospitalized, the patient evolved with a progressive improvement of symptoms with subsequent discharge from the hospital. However, the follow-up visits were scheduled in another institution, and the patient was lost to follow up.

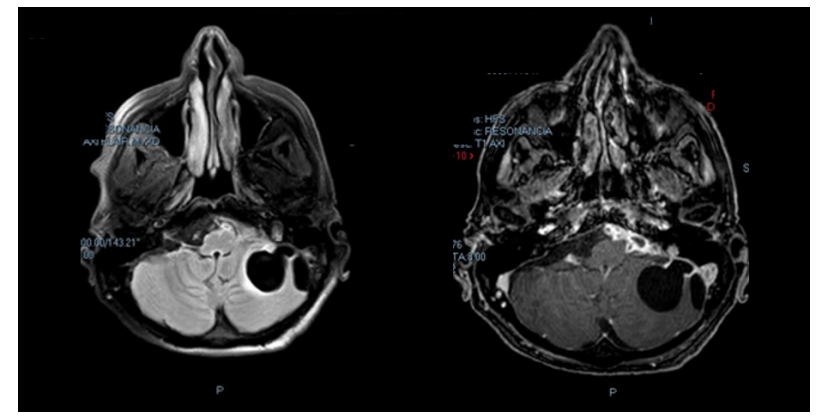

Figure 2 - Axial Magnetic Resonance imaging of the brain. Flair (left) and T1 contrast (right) images with a multicystic lesion located in the subarachnoid space at the level of the lateral surface of the left cerebellar hemisphere. Note the absence of a scolex and the "mass effect".

\section{DISCUSSION}

Cysticerci are small vesicles that consist of the vesicular walls and the scolex. However, in some cysticercus the scolex cannot be identified because these parasites are composed of membranes attached to each other that tend to group in clusters resembling grapes. This form is called the racemose form of cysticerci and is observed in parasites located within the cerebrospinal fluid (CSF) cisterns at the base of the brain as the case of our patient ${ }^{10}$. 


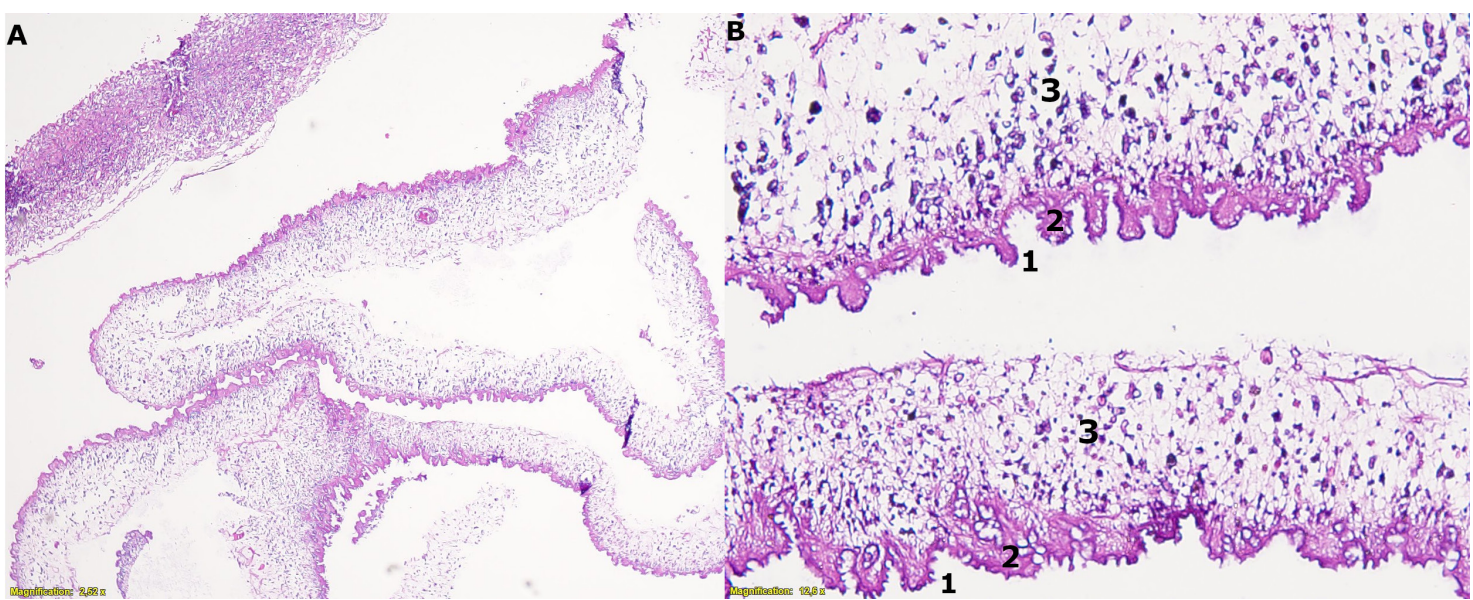

Figure 3 - Hematoxylin-Eosin staining of a biopsy specimen from the cerebellar cyst: A) Upper left image. Outer edge of the cyst containing an intense inflammatory infiltrate. Larger image. Wall of a cysticercus cyst in small magnification (2.52 x); B) Wall of a cysticercus cyst with cuticular (1), cellular (2), and reticular (3) layer (12.6 x). The cuticular layer shows small, villous protrusions, the cellular layer is located just below the cuticular layer, and the reticular layer is the larger one, containing a loosely arranged matrix, and excretory canaliculi.

Subarachnoid NC has a poor prognosis and a high mortality rate ranging from $20 \%$ to $50 \%{ }^{7,8}$ in part due to the risk of developing cysts that can obstruct the drainage of cerebrospinal fluid leading to a high intracranial pressure and, if severe enough, subsequent hydrocephaly will take place possibly leading to death ${ }^{7,11}$. In the case presented here, a ventriculoperitoneal shunt was performed to prevent further complications.

Our patient was taken to the emergency room due to headaches, gait alterations and memory loss with behavioral changes; nevertheless, the clinical course is often varied depending on the location and the size of the cyst, but tends to be chronic in nature ${ }^{4,7}$. Intracranial hypertension can be found in $75 \%$ of the cases and manifests as headaches in almost $50 \%$ of the patients, cognitive impairment is seen in up to $21 \%$ of the cases, but the prevalence of cerebellar symptoms is not known due the scarcity of cases reported in the literature ${ }^{8,9}$. Furthermore, these symptoms are not specific which makes the diagnosis difficult and implies a challenge for the clinician ${ }^{7,11}$.

Radiologic studies such as CT or MRI are useful for diagnosis, classification and treatment monitoring in $\mathrm{NC}^{3}$. Nevertheless, there is no direct correlation between the presence of intracranial lesions in radiological studies and the clinical presentation, delaying the diagnosis ${ }^{4}$. The most common finding is hydrocephalus caused by an inflammatory occlusion secondary to a fibrous arachnoiditis. Cystic subarachnoid lesions may be small or reach a large size if they are located within the basal CSF cisterns where they usually have a multilobulated appearance behaving as a mass growing lesion as in our case $^{10}$. However, subarachnoid NC represents an entity with a difficult radiological diagnosis compared to its parenchymal counterpart because parasites emit a signal similar to the one of the cerebrospinal fluid in MRI which limits their visualization ${ }^{11,12}$. In addition, in the racemose form, the scolex cannot be identified. The mechanism responsible is not well understood, but the disappearance of the scolex in the racemose form could be secondary to a degenerative process called hydropic degeneration, that in turn will favor the continuous entrance of CSF inside the vesicle ${ }^{10}$. Racemose neurocysticercosis with cerebellar involvement has been rarely reported probably as a result of a reduced blood flow in comparison with the cerebrum ${ }^{8}$.

Concerning the serological diagnosis, tests with enzymelinked immunoelectrotransfer blot (EITB) are recommended as a confirmatory test, with $100 \%$ specificity and $50-98 \%$ sensitivity. However, EITB is not widely available, and a positive result is not necessarily pathognomonic of NC. On the other hand, the detection of antigens by Enzymelinked immunosorbent assay (ELISA) is not recommended due to its low sensitivity and specificity ${ }^{13}$. For this reason, EITB is the most useful laboratory tool when there is an unclear diagnosis ${ }^{13}$. In this case, the test was available and was performed during the patient's second visit. However, while waiting for the results, a clinical deterioration forced a brain biopsy.

Diagnosis of subarachnoid NC via CSF analysis can be challenging because of its similarity to other infectious diseases, like tuberculosis or subarachnoid involvement of systemic malignancies that may have comparable clinical and imaging findings ${ }^{14,15}$. To help in the diagnosis of NC, Del Brutto ${ }^{10}$ established the new diagnostic criteria based on imaging studies and clinical findings.

Cysticide drugs have been used in subarachnoid NC since 1990 and subsequent non-controlled studies reported 
the efficacy of albendazole for destroying subarachnoid cysticercus. However, there are important differences in the dose used, the duration, and the use of combined therapy with praziquantel ${ }^{16}$. Nevertheless, the 2017 Clinical Practice Guidelines by the Infectious Diseases Society of America recommends the use of Albendazole ( $15 \mathrm{mg} / \mathrm{kg} /$ day) in combination with Praziquantel $\mathrm{l}^{13}$ but in many countries such as Colombia, Praziquantel is not commercially available, and an alternative therapeutic approach is needed due to the risk of therapeutic failure. To date there are only two studies that compared the usual dose of albendazole $(15 \mathrm{mg} / \mathrm{kg} / \mathrm{day})$ with a higher dose ( $30 \mathrm{mg} / \mathrm{kg} / \mathrm{day})$, showing promising results, unfortunately, both studies were small, and the treatment duration was heterogenous ${ }^{17,18}$. In our patient an immediate clinical improvement seemed to be an indicator of adequate tolerance, unfortunately the patient was lost to follow-up.

It is important to mention that there are also case reports of the parasite persistence despite the use of albendazole. This could be in part due to cysticide drugs differentially affecting the parasites according to their development stage, i.e., the lesser the penetration in the subarachnoid space, the higher the variability of plasma and CSF levels due to individual differences in the bioavailability. The use of corticosteroids could act as a key component of the immunological control of the infection ${ }^{19}$. Before initiating the therapy, the management of intracranial hypertension and hydrocephalus is recommended due to the risk of worsening of the intracranial hypertension with the use of cysticide drugs ${ }^{13}$. Surgical management is limited to the patients in whom medical treatment is not effective or feasible ${ }^{13,20}$.

To our knowledge, treatment for subarachnoid NC has not yet been clearly been established and the duration of the treatment is not known ${ }^{13,16}$.

\section{CONCLUSION}

This case report highlights the diagnostic challenges that subarachnoid $\mathrm{NC}$ represents and the gaps in knowledge regarding the optimum way to treat these patients. In countries with high prevalence of cysticercosis, like Colombia, it is important to include this entity in the differential diagnosis of chronic meningitis and cystic lesions in the cerebellum. This case should encourage the design of clinical studies to address important questions about the true epidemiology of this entity, the clinical features that could help in its prompt identification and the best therapeutic strategies. For an endemic disease in many South American countries, there is much we still do not know.

\section{CONFLICT OF INTERESTS}

None of the authors declares conflict of interests.

\section{FUNDING}

None of the authors declares financial support.

\section{REFERENCES}

1. Garcia HH, Nash TE, Del Brutto OH. Clinical symptoms, diagnosis, and treatment of neurocysticercosis. Lancet Neurol. 2014;13:1202-15

2. Sanchez-Larsen A, Monteagudo M, Lozano-Setien E, GarciaGarcia J. Neurocisticercosis racemosa subaracnoidea gigante y ventricular: a propósito de un caso. Rev Argent Microbiol. 2015;47:201-5.

3. Bazan R, Hamamoto Filho PT, Luvizutto GJ, Nunes HR, Odashima NS, Santos AC, et al. Clinical symptoms, imaging features and cyst distribution in the cerebrospinal fluid compartments in patients with extraparenchymal neurocysticercosis. PLoS Negl Trop Dis. 2016;10:e005115.

4. Sierra MM, Arroyo M, Torres MC, Cruz NR, Hernández FG, Taboada D, et al. Extraparenchymal neurocysticercosis: demographic, clinicoradiological, and inflammatory features. PLoS Negl Trop Dis. 2017;11:e0005646.

5. Flórez-Sánchez AC, Pastrán-Sandra M, Vargas NS, Beltrán M, Enriquez Y, Peña AP, et al. Cisticercosis en Colombia: estudio de seroprevalencia 2008-2010. Acta Neurol Colomb. 2013;29:73-86.

6. Qi B, Ge P, Yang H, Bi C, Li Y. Spinal intramedullary cysticercosis: a case report and literature review. Int J Med Sci. 2011;8:420-3

7. Fleury A, Carrillo-Mezo R, Flisser A, Sciutto E, Corona T. Subarachnoid basal neurocysticercosis: a focus of the most severe form of disease. Expert Rev Anti Infect Ther. 2011;9:123-33.

8. Kim SW, Kim MK, Oh SM, Park SH. Racemose cysticercosis in the cerebellar hemisphere. J Korean Neurosurg Soc. 2010;48:59-61.

9. Zhu L, Shi Y, Pan X, Mo L, Weng X. Successful treatment of isolated cerebellar cysticercosis with albendazole. Chin Med J (Eng). 2003;116:637-8

10. Del Brutto OH. Neurocysticercosis. In: Biller J, Ferro JM, editors. Handbook of clinical neurology, vol. 121: neurologic aspects of systemic disease, part III. Amsterdam: Elsevier; 2014. p.1445-59.

11. Cure GC, Roldán NG, Quiñones GP, Acosta AD, Bermúdez S, Hakim D, et al. Neurocisticercosis. Acta Neurol Colomb. 2009;25:42-53.

12. Zhao JL, Lerner A, Shu Z, Gao XJ, Zee CS. Imaging spectrum of neurocysticercosis. Radiol Infect Dis. 2015;1:94-102. 
13. White AC Jr, Coyle CM, Rajshekhar V, Singh G, Hauser A, Mohanty A, et al. Diagnosis and treatment of neurocysticercosis: 2017 Clinical Practice Guidelines by the Infectious Diseases Society of America (IDSA) and the American Society of Tropical Medicine and Hygiene (ASTMH). Clin Infect Dis. 2018;66:e49-75.

14. Nash TE, O'Connell EM, Hammoud DA, Wetzler L, Ware JM, Mahanty S. Natural history of treated subarachnoid neurocysticercosis. Am J Trop Med Hyg. 2020;102:8-89.

15. Gripper LB, Welburn SC. Neurocysticercosis infection and disease: a review. Acta Trop. 2017;166:218-24.

16. Del Brutto $\mathrm{OH}$. Current approaches to cysticidal drug therapy for neurocysticercosis. Expert Rev Anti Infect Ther. 2020;18:78998.
17. Göngora-Rivera F, Soto-Hernández JL, González-Esquivel D, Cook HJ, Márquez-Caraveo C, Hernández-Dávila R, et al. Albendazole trial at 15 or $30 \mathrm{mg} / \mathrm{kg} /$ day for subarachnoid and intraventricular cysticercosis. Neurology. 2006;66:436-8.

18. Márquez-Caraveo C, Góngora-Rivera F, Santos-Zambrano J, Hernández R, Soto-Hernández JL. Pre-treatment with corticosteroids and a single cycle of high dose albendazole for subarachnoidal cysticercosis. J Neurol Neurosurg Psychiatry. 2004;75:938-9.

19. Cárdenas G, Carrillo-Mezo R, Jung H, Sciutto E, Hernandez JL, Fleury A. Subarachnoidal neurocysticercosis non-responsive to cysticidal drugs: a case series. BMC Neurol. 2010;10:16.

20. Garcia HH. Neurocysticercosis. Neurol Clin. 2018;36:851-64. 